\title{
IMMERSIVE ENVIRONMENT TECHNOLOGIES FOR PLANETARY EXPLORATION WITH APPLICATIONS FOR MIXED REALITY
}

\author{
John Wright, Frank Hartman, Brian Cooper \\ Jet Propulsion Laboratory
}

\begin{abstract}
Immersive environments are successfully being used to support mission operations at the Jet Propulsion Laboratory. This technology contributed to the Mars Pathfinder Mission in planning sorties for the Sojourner rover. Results and operational experiences with these tools are being incorporated into the development of the second generation of mission planning tools. NASA's current plan includes two rovers being deployed to Mars in 2003, landing in early 2004. The next generation Rover Sequencing and Visualization Program (RSVP) utilizes existing technologies from Pathfinder, and more, to provide a multimodal, collaborative, partially immersive environment for mission planning. This system includes tools for planning long range sorties for highly autonomous rovers, tools for building the threedimensional (3D) models of the terrain being explored, and advanced tools for visualizing telemetry from remote spacecraft and landers. These tools comprise a system for immersing the operator in the environment of another planet, body, or space to make the mission planning function more intuitive and effective. This paper describes the set of tools that will be utilized to provide the immersive capability within the RSVP, including terrain modelling, visualization with overlaid iconosymbology, simulation and rehearsal capability, and integration of actual rover telemetry. Applications of these technologies to mixed reality environments are discussed.
\end{abstract}

Key words: Immersive environments, Pathfinder, terrain modelling, visualization, Mars, Rover Control Workstation, mixed reality, JPL.

The original version of this chapter was revised: The copyright line was incorrect. This has been corrected. The Erratum to this chapter is available at DOI: 10.1007/978-0-387-35660-0_65 


\section{Introduction}

The application of immersive environments to mission operations within the Visualization and Earth Sciences Applications Group at the Jet Propulsion Lab began with the Sense of Active Presence (SOAP) task. The group has been involved in scientific data visualization for a variety of missions. The SOAP task was the first to apply the terrain modelling and animation techniques developed within the group in a rapid turnaround mode to support mission operations. During the Mars Pathfinder mission, tools developed within the SOAP task produced 3D models of the terrain around the lander for use in generating and reviewing operations sequences for the Sojourner rover.

Current tasks within the group are extending the Rover Control Workstation (RCW) used during Pathfinder to incorporate 3D visualization in a more integrated manner. These tasks are building on systems described in their early stages in previous work [1] in which early research into immersive systems hardware and software were described. A new task is the System for Unifying Multiresolution Models and Integrating Threedimensional Terrains (SUMMITT) task which is integrating various subsystems into a more complete system for creating the 3D terrain models from orbiter, descent, lander, and rover imagery. These terrain models will be used to support the Mars '03 rover missions and landed operations. The Advanced Telemetry Visualization (ATV) task uses 3D visualization for reviewing mission operations through playback of telemetry returned from the craft and will also be used to support Mars '03. The RSVP is an upgraded version of the RCW with more robust sortie planning and visualization tools. These include multimodal visualization tools offering a map view, a stereo view, and an immersive $3 \mathrm{D}$ view, as well as sortie rehearsal tools utilizing terrain models and mission playback from telemetry using ATV.

\section{RCW Architecture}

Programming the rover to perform a sortie can be a laborious process as obstacles must be analyzed and the safest route selected. Control-feedback loop turnaround times in minutes or hours preclude direct control of a rover in a man-in-the-loop configuration. In additon, bandwidth limits on the data that can be downlinked from Mars preclude anything like video. The Mars '03 rovers will roam multiple kilometers from their landing sites. To support this capability, rovers will include onboard intelligence to perform a higher level of sortie planning, navigation, and obstacle avoidance than has been possible to date. This new paradigm of rover functionality will call for a 
new type of operator control, with much more interaction with the environment and less low-level control of rover operations. This new modality will utilize data visualization technology to provide a multidimensional "sense of presence" for operation and data analysis of teleoperated and semiautonomous vehicles. This will give the operator, or mission planner, the freedom to roam the vehicle's environment, thus providing a more intuitive and richer human/computer interface. The operator will be able to explore potential sites through the interface prior to committing the rover. Routes and destinations can be preexamined and designated through the interface. This adaptation of commercial technologies to the flight system environment provides a more sophisticated and intuitive interface for human interaction with exploration vehicles and provides a better understanding of in-situ operations leading to better decision making. An additional benefit is the use of the immersive interface for analysis and interaction with the science data, both by researchers and by the educational community and the public.

Both the vehicle and the operator station begin with an initial database describing the terrain to be explored. This database will typically be created with descent imagery and lander panoramic data merged with previously available data from orbiters or Earth-based sensors. The panoramic imagery from the lander or rover is the main science data being returned to Earth. This imagery, captured with stereo cameras, is used to build a visual database representing the terrain surrounding the rover as described in the next section. The operator will be immersed in the visual database, exploring the sortie area, selecting interesting areas to explore, and designating desired routes. The rover will receive this planning information, comparing potential routes with its terrain database to identify hazards, and select a route. The vehicle will traverse the route, using alternative routes as appropriate to avoid previously unknown hazards, and transmit imagery and other data back to the ground station. As new data is received, the visual database is updated, essentially filling in the coarse picture with high-resolution information. Any part of the visual database may be explored at any time and multiple individuals may access the database at the same time. This allows researchers and educators access to data in parallel with the operators.

The RCW deployed for the Pathfinder mission was based on two basic visualization tools, the Stereo View and the Flying Camera View as described in [2]. These two basic modalities are continued in the updated version, RSVP, and combined with a Map View tool to provide the most important visualization functions of the in-situ environment. The Stereo View mode provides the most basic, raw look at the image data returned from the stereo imagers. The imagery is displayed using a stereo monitor with the individual stereo pairs arranged in position relative to the camera 
pointing when the images were captured. The Flying Camera tool alleviates the problem of judging the separation of objects in the foreground and background by providing a means to examine the in-situ environment from any vantage point. The stereo imagery from the imagers is processed to generate a 3D model of the terrain in the immediate vicinity. This model is stored in a form that can be loaded and visualized with a high level of detail and interactive rendering rates. The virtual camera can then be positioned to view possible routes and constrictions to verify that the rover can indeed traverse the planned route. The Flying Camera tool also supports visualization of a model of the rover that can be positioned anywhere within the environment to verify fit and feasibility. The Map View tool adds a natural, maplike visualization mode to the suite of tools in the RCW. It provides a bird's-eye view of the sortie area and natural access to georeferencing information and to navigation data such as landmark datasets, reference points, and direction. Other features of the Map View tool include specification of hazard or protected regions and contour lines for analysis of slope.

The other main component of the RSVP for sortie planning is the Activity Editor which is essentially a text visualization tool for displaying the sequence of commands being produced. All four tools are integrated with a message passing executive which maintains a consistent view of the planned sequence among all the tools. Commands, such as traverse to waypoint, may be specified in any of the tools and the creation or editing of such a command is immediately reflected in the other tools. Additionally, multiple copies of each visualization tool may be launched by the same executive yet running on distributed systems to provide for a collaborative planning environment.

\section{Modeling Terrains}

The System for Unifying Multiresolution Models and Integrating Threedimensional Terrains (SUMMITT) task has the goal of developing the underlying modelling technology for supporting missions involving rovers. Three-dimensional models of terrain areas are an invaluable asset in planning operations and in reviewing the predicted and telemetered operations of a robot arm. The SUMMITT task had the initial goal of supporting the Mars Volatiles and Climate Surveyor (MVACS) team during the Mars ' 98 mission, which unfortunately failed. The next Mars surface operations missions are the Mars ' 03 missions that plan to land two rovers. These missions expect to have orbital imagery from Mars Global Surveyor (MGS) and Mars Odyssey, descent imagery from the lander, and lander 
imagery from a stereo imager similar to the Imager for Mars Pathfinder (IMP) used during the Pathfinder mission. These sources of imagery will be combined to create a multiresolution terrain model with very high resolution detail available within the immediate area of operations of the rover.

The three primary sources of data are orbiter imagery, descent imagery, and lander imagery. Each type of imagery is partially processed independently, then combined with data derived from the other imagery to create the multiresolution terrain models. The fundamental problem is the registration of the different terrain models generated independently from the different data sources. The primary method which has been demonstrated and selected at JPL is $3 \mathrm{D}$ registration of the $3 \mathrm{D}$ models. These techniques use optimization techniques to match the 3D surface of the terrain model generated from the lander imagery to a similar 3surface generated from the baseline model. The resulting match parameters are then used to correct the position and orientation information for the lander. Our approach uses volumetric primitives (voxels) to represent the terrain to be matched. Voxels have some advantages over the polygonal surface matching methods in that it is easier to represent unknown volumes, such as regions occluded by rocks and hills, and easy to use, multiresolution data structures are available in which to combine the models once they are matched. The entire voxel model is stored in an octree structure which supports multiresolution data and rapid access while utilizing significantly less memory than a three-dimensional grid.

Once a terrain model has been generated, it is typically converted to a polygon model for utilization by a visualization tool. The primary requirements here are for high fidelity in the terrain imagery with high rendering performance. The multiresolution nature of the data precludes the use of a simple algorithm such as Marching Cubes [3] for converting to a polygon surface. To extract a simple polygon model from the octree, a modified version of the Marching Triangles algorithm [4], was developed. The modified version utilizes most of the points in the model to generate a Delaunay triangulation in three-space. An additional tool is under development to convert the models to an Active Surface Definition (ASD) model for use in high performance renderers utilizing Open Performer.

\section{Applications to Mixed Reality}

The terrain modelling tools described above have several applications to virtual and mixed reality systems. Similar work has been described in [5] and [6] on the use of the ICP algorithm for registering multiple range images to generate detailed 3D models of objects for digital archival and virtual 
exploration. The key difference in our approach has been embracing multiresolution data as fundamental to our activities. While fairly standard in the flight simulator area, it is new for using range images to create models. The terrain models generated by our system will have wide applicability for visualization in immersive systems for science operations and mobility planning for the rovers.

Of higher interest is the utilization of similar techniques during a mixed reality session. One of the key problems in mixed and augmented reality systems is the registration of the virtual and the real worlds. Approaches using head trackers for indoor systems or GPS-type position sensors for outdoor applications have been widely used. So far, these approaches have suffered from noise in the position and orientation information that give rise to jitter in the visual system. Another approach is to use imagery of the scene to compute the position and orientation of the user. This may be done using a single imager with feature recognition capability. The recognition of features may be aided by the use of markers in the environment.

In a large gaming area, however, the use of markers may be precluded and the actual feature appearance may not be well known. Such is the case with the areas of Mars being explored. In this case, stereo imagery may be utilized to model the local topology of the environment. Then the local model may be registered with a global model, using position estimates, tracker input, or GPS data as a seed, to determine a reasonable alignment and thus localizing the viewer in position and orientation. This method has the advantage that it is relatively illumination independent since it relies on the shape of the models for the registration. Our use of the ICP algorithm for this type of data has also shown that the resolution of the two datasets need not be the same. This is important for cases where the global model is built from data collected by a remote sensor while the local model is built from data collected by an in-situ sensor. The ICP algorithm can register data with at least one order of magnitude difference in resolution. Two orders of magnitude can become problematic for terrain models but may be workable for structures and other models with walls, corners, and other distinct features. For Mars, we anticipate that we will have models of the landing site built from orbital imagery at one meter resolution while the rover imagery may be used to generate models with one centimeter resolution. We hope to use imagery captured during descent to assist in bridging this gap and improving model registration. 

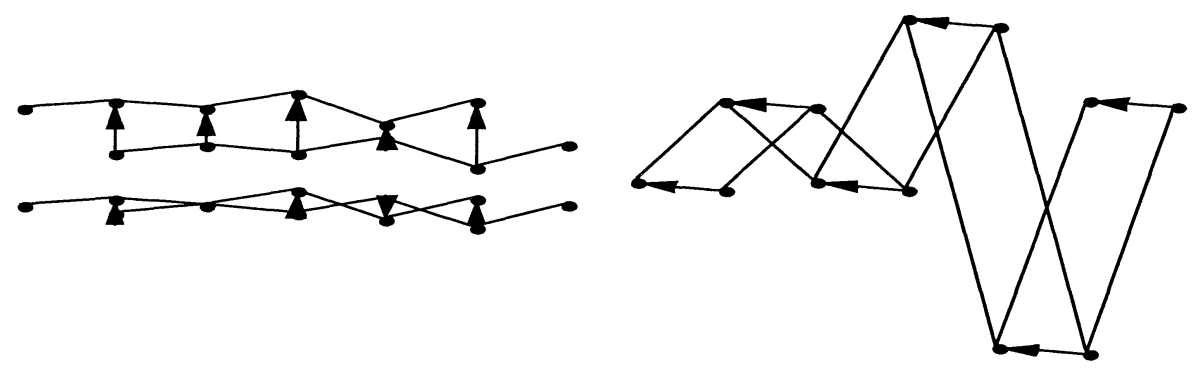

Figure 1. Figure 1 - Effect of Z-stretch on Point Matching

Our implementation of the ICP algorithm is certainly not fast enough to perform model registration in realtime nor is that a requirement for our application. However, utilization of octrees during the registration process does aid in improving performance when searching for the nearest point in the match set and could be an important tool for localization in mixed reality applications. Another feature that aids the registration process and may have applicability to such applications is a "Z-stretch" in which the model is scaled along the $\mathrm{z}$ axis. When processing terrain models, the horizontal resolution may be larger than the vertical feature size. This causes a problem, particularly for gridded elevation models in which the alignment of the grid has more weight than the misalignment of the vertical features. In this case, the closest point to the model will often be the nearest grid point. This grid alignment can produce local minima that affect the final solution. One method to address this problem involves easing the constraints on horizontal alignment to give more weight to the vertical features. The solution that we have applied is to stretch the models along the $\mathrm{z}$ axis to enhance the vertical features as shown in Figure 1. The scaling is applied as we build the octree to hold the global model. Then the local model is also scaled as we perform the search for the closest point in the global model. The scaling is part of our overall transformation process and adds no additional processing time.

\section{Conclusions}

The Jet Propulsion Lab is moving forward with several efforts to develop enhanced immersive technologies for supporting mission operations. From the highly successful Mars Pathfinder mission through upcoming Mars missions launching in 2003, immersive technologies and systems will aid the operations teams in making mission critical decisions. Creating models of 
the operational environment and providing visualization tools to explore and interact with that environment are the key aspects being explored. This will be especially important as exploratory craft gain in autonomy and intelligence and begin to explore farther afield from their initial landing site. While the level of immersion can vary from simple two-dimensional displays through stereo displays and virtual workbenches and on up to full immersion with head-mounted displays, haptic and tactile feedback [7], and intuitive manipulators [8], the main goal is to provide the operators with a better understanding of the operational environment.

\section{Acknowledgements}

The work described in this paper was performed at the Jet Propulsion Laboratory, California Institute of Technology, under a contract with the National Aeronautics and Space Administration.

\section{References}

[1] Wright, J., Hartman, F., and Cooper, B. (1998) Immersive Environments for Mission Operations: Beyond Mars Pathfinder, Proceedings of SpaceOps '98, Tokyo, Japan, 1998.

[2] Cooper, B. (1998) Driving on the Surface of Mars Using the Rover Control Workstation, Proceedings of SpaceOps '98, Tokyo, Japan, June, 1998.

[3] Lorensen, W. and Cline, H. (1987) Marching Cubes: A High Resolution 3D Surface Construction Algorithm. Proceedings of SIGGRAPH '87, pp. 163-169, July 1987.

[4] Hilton, A., Stoddart, A.J., Illingworth, J., and Windeatt, T. (1996) Marching Triangles: Range Image Fusion for Complex Object Modelling. Proceedings of International Conference on Image Processing, 1996.

[5] Nishino, K. \& Katsushi, I. (2002) Robust Simultaneous Registration of Multiple Range Images. Proceedings of the \%th Asian Conference on Computer Vision, Melbourne, Australia, January 2002.

[6] Miyazaki, D., Ooishi, T., Nishikawa, T., Sagawa, R., Nishino, K., Tomomatsu, T., Takase, Y., and Katsushi, I., The Great Buddha Project: Modelling Cultural Heritage through Observation. Proceedings of the 6th International Conference on Virtual Systems and Multimedia, Gifu, Japan, October 2000,

[7] Hughes, R.G. \& Forrest, A.R. (1996). Perceptualisation Using a Tactile Mouse. IEEE Visualization '96 Proceedings Piscataway, NJ: IEEE. 181-188.

[8] Shimoga, K. B. (1993). A Survey of Perceptual Feedback Issues in Dexterous Telemanipulation. VRAIS '93: 1993 IEEE Virtual Reality Annual International Symposium. Piscataway, NJ: IEEE. 263-279. 